\title{
ON KOSTANT'S PARTIAL ORDER ON HYPERBOLIC ELEMENTS
}

\author{
HUAJUN HUANG AND SANGJIB KIM
}

\begin{abstract}
We study Kostant's partial order on the elements of a semisimple Lie group in relations with the finite dimensional representations. In particular, we prove the converse statement of [3, Theorem 6.1] on hyperbolic elements.
\end{abstract}

A matrix in $\mathrm{GL}_{n}(\mathbb{C})$ is called elliptic (resp. hyperbolic) if it is diagonalizable with norm 1 (resp. real positive) eigenvalues. It is called unipotent if all its eigenvalues are 1 . The complete multiplicative Jordan decomposition of $g \in \mathrm{GL}_{n}(\mathbb{C})$ asserts that $g=e h u$ for $e, h, u \in \mathrm{GL}_{n}(\mathbb{C})$, where $e$ is elliptic, $h$ is hyperbolic, $u$ is unipotent, and these three elements commute (cf. [2, p430-431]). The decomposition can be easily seen when $g$ is in a Jordan canonical form: if the diagonal entries (i.e. eigenvalues) of the Jordan canonical form are $z_{1}, \cdots, z_{n}$, then

(1) $\quad e=\operatorname{diag}\left(\frac{z_{1}}{\left|z_{1}\right|}, \cdots, \frac{z_{n}}{\left|z_{n}\right|}\right), \quad h=\operatorname{diag}\left(\left|z_{1}\right|, \cdots,\left|z_{n}\right|\right)$,

and $u=h^{-1} e^{-1} g$ is an upper triangular matrix with diagonal entries 1.

The above decomposition can be extended to semisimple Lie groups. Let $G$ be a connected real semisimple Lie group with Lie algebra $\mathfrak{g}$. An element $e \in G$ is elliptic if Ad $e \in$ Aut $\mathfrak{g}$ is diagonalizable over $\mathbb{C}$ with eigenvalues of modulus 1 . An element $h \in G$ is called hyperbolic if $h=\exp X$ where $X \in \mathfrak{g}$ is real semisimple, that is, ad $X \in$ End $\mathfrak{g}$ is diagonalizable over $\mathbb{R}$ with real eigenvalues. An element $u \in G$ is called unipotent if $u=\exp X$ where $X \in \mathfrak{g}$ is nilpotent, that is, ad $X \in$ End $\mathfrak{g}$ is nilpotent. The complete multiplicative Jordan decomposition [3, Proposition 2.1] for $G$ asserts that each $g \in G$ can be uniquely written as

$$
g=e h u,
$$

2000 Mathematics Subject Classification: Primary 22E46

Key Words: Semisimple Lie groups, Complete multiplicative Jordan decomposition, Hyperbolic elements, Majorizations. 
where $e$ is elliptic, $h$ is hyperbolic and $u$ is unipotent and the three elements $e, h, u$ commute. We write $g=e(g) h(g) u(g)$.

When $G=\mathrm{SL}_{n}(\mathbb{C})$, the above decomposition in $G$ coincides with the complete multiplicative Jordan decomposition in $\mathrm{GL}_{n}(\mathbb{C})$ defined in the first paragraph. Let us elaborate on it. Given $g \in \mathrm{SL}_{n}(\mathbb{C})$, there exists $y \in \mathrm{SL}_{n}(\mathbb{C})$ such that $y g y^{-1} \in \mathrm{SL}_{n}(\mathbb{C})$ is in a Jordan canonical form. Since the complete multiplicative Jordan decompositions of $g$ in both $\mathrm{GL}_{n}(\mathbb{C})$ and $\mathrm{SL}_{n}(\mathbb{C})$ are preserved by conjugations in $\mathrm{SL}_{n}(\mathbb{C})$, without lost of generality, we may assume that $g \in \mathrm{SL}_{n}(\mathbb{C})$ is already in a Jordan canonical form with diagonal entries $z_{1}, z_{2}, \cdots, z_{n}$. Then the complete multiplicative Jordan decomposition of $g$ in $\mathrm{GL}_{n}(\mathbb{C})$ is $g=e h u$, where $e$ and $h$ are given in (1) and $u=h^{-1} e^{-1} g$ is an upper triangular matrix with diagonal entries 1 . Let $E_{i j} \in \mathfrak{g l}_{n}(\mathbb{C})$ denote the matrix with 1 in the $(i, j)$-entry and 0 elsewhere. Then with respect to the following basis of $\mathfrak{s l}_{n}(\mathbb{C})$ :

$$
\left\{E_{i j} \mid i \neq j, 1 \leq i, j \leq n\right\} \cup\left\{E_{i i}-E_{(i+1)(i+1)} \mid 1 \leq i \leq n-1\right\},
$$

the matrix Ad $e$ is diagonal with norm 1 eignevalues $z_{i} z_{j}^{-1} /\left|z_{i} z_{j}^{-1}\right|(i \neq$ $j$ ) and 1. This shows that $e$ is elliptic in $\mathrm{SL}_{n}(\mathbb{C})$. Clearly, $h=\exp X$ for a real semisimple element $X:=\log h \in \mathfrak{s l}_{n}(\mathbb{C})$, and $u=\exp Y$ for a nilpotent element

$$
Y:=\log u=-\frac{I_{n}-u}{1}-\frac{\left(I_{n}-u\right)^{2}}{2}-\cdots-\frac{\left(I_{n}-u\right)^{n-1}}{n-1} \in \mathfrak{s l}_{n}(\mathbb{C}),
$$

where $I_{n}$ is the identity matrix. This shows that $h$ (resp. $u$ ) is hyperbolic (resp. unipotent) in $\mathrm{SL}_{n}(\mathbb{C})$. Thus $g=e h u$ is also the complete multiplicative Jordan decomposition of $g$ in $\mathrm{SL}_{n}(\mathbb{C})$.

Let us return to a connected real semisimple Lie group $G$ with Lie algebra $\mathfrak{g}$. Fix a Cartan decomposition $\mathfrak{g}=\mathfrak{k} \oplus \mathfrak{p}$ (e.g., [2]), and let $\mathfrak{a} \subseteq \mathfrak{p}$ be a maximal abelian subalgebra. Let $A:=\exp$ a. Then the hyperbolic component $h(g)$ of $g$ is conjugate to some element $a(g) \in A$. See, for example, [3, Proposition 2.4]. We denote

$$
A(g):=\exp (\operatorname{conv}(W \cdot \log a(g))),
$$

where $W$ is the Weyl group of $(\mathfrak{g}, \mathfrak{a})$ and $\operatorname{conv}(W \cdot \log a(g))$ is the convex hull of the Weyl group orbit of $\log a(g)$ in $\mathfrak{a}$.

Kostant defined a partial order on the elements of $G$ as

$$
g_{1} \geq g_{2} \Longleftrightarrow A\left(g_{1}\right) \supseteq A\left(g_{2}\right),
$$

and then established the following results: 
Theorem 1. [3, Theorem 3.1] Let $g_{1}, g_{2} \in G$. Then $g_{1} \geq g_{2}$ if and only if $\left|\pi\left(g_{1}\right)\right| \geq\left|\pi\left(g_{2}\right)\right|$ for all finite dimensional representations $\pi$ of $G$ where $|\cdot|$ denotes the spectral radius.

Theorem 2. [3, Theorem 6.1] Let $h_{1}, h_{2} \in G$ be hyperbolic. Write $\chi_{\pi}$ for the character of a representation $\pi$ of $G$. If $h_{1} \geq h_{2}$, then $\chi_{\pi}\left(h_{1}\right) \geq \chi_{\pi}\left(h_{2}\right)$ for all finite dimensional representations $\pi$ of $G$.

In [3, Remark 6.1], Kostant asked if the converse of Theorem 2 is true or not. Our goal is to answer this question affirmatively and thus we have the following result:

Theorem 3. Let $h_{1}$ and $h_{2}$ be two hyperbolic elements in a connected real semisimple Lie group $G$. Then $h_{1} \geq h_{2}$ if and only if $\chi_{\pi}\left(h_{1}\right) \geq$ $\chi_{\pi}\left(h_{2}\right)$ for all finite dimensional representations $\pi$ of $G$.

Before proving the above result, we need the following two lemmas.

Lemma 4. Let $C$ and $D$ be two diagonal matrices in $\mathrm{GL}_{n}(\mathbb{C})$ with positive diagonal entries. If the spectral radii $|C|>|D|$, then there exists a finite dimensional representation $\pi$ of $\mathrm{GL}_{n}(\mathbb{C})$ such that $\chi_{\pi}(C)>$ $\chi_{\pi}(D)$.

Proof. Let $\pi_{m}$ be the representation of $\mathrm{GL}_{n}(\mathbb{C})$ on the $m$-th symmetric power of the natural representation $\operatorname{Sym}^{m}\left(\mathbb{C}^{n}\right)$. Then, the character $\chi_{m}$ of $\pi_{m}$ takes the following value on $\operatorname{diag}(x)=\operatorname{diag}\left(x_{1}, x_{2}, \cdots, x_{n}\right) \in$ $\mathrm{GL}_{n}(\mathbb{C})$ :

$$
\chi_{m}(\operatorname{diag}(x))=\sum_{\substack{\ell_{1}, \ell_{2}, \cdots, \ell_{n} \geq 0 \\ \ell_{1}+\cdots+\ell_{n}=m}} \prod_{i=1}^{n} x_{i}^{\ell_{i}}
$$

So $\chi_{m}(\operatorname{diag}(x))$ is the sum of all $\left(\begin{array}{c}m+n-1 \\ n-1\end{array}\right)$ monomials of $x_{1}, \cdots, x_{n}$ of degree $m$. Suppose $c:=|C|>|D|=: d>0$. Because $\lim _{m \rightarrow \infty}(m+n)^{n / m}=1$, there exists a sufficiently large $m$ such that

$$
\frac{c}{d}>(m+n)^{n / m}
$$


Write $C:=\operatorname{diag}\left(c_{1}, \cdots, c_{n}\right)$ and $D:=\operatorname{diag}\left(d_{1}, \cdots, d_{n}\right)$. Then

$$
\begin{aligned}
\chi_{m}(C) & =\sum_{\substack{\ell_{1}, \ell_{2}, \cdots, \ell_{n} \geq 0 \\
\ell_{1}+\cdots+\ell_{n}=m}} \prod_{i=1}^{n} c_{i}^{\ell_{i}} \\
& \geq c^{m}>(m+n)^{n} d^{m}>\left(\begin{array}{c}
m+n-1 \\
n-1
\end{array}\right) d^{m} \\
& \geq \sum_{\substack{\ell_{1}, \ell_{2}, \cdots, \ell_{n} \geq 0 \\
\ell_{1}+\cdots+\ell_{n}=m}} \prod_{i=1}^{n} d_{i}^{\ell_{i}}=\chi_{m}(D) .
\end{aligned}
$$

This completes the proof.

The following results are shown in [3, Proposition 3.4 and its proof]: for a representation $\pi: G \rightarrow \mathrm{GL}_{n}(\mathbb{C})$,

- If $u \in G$ is unipotent, then all the eigenvalues of $\pi(u)$ are equal to 1 ;

- If $h \in G$ is hyperbolic in $G$, then $\pi(h)$ is diagonalizable and all the eigenvalues of $\pi(h)$ are positive;

- If $e \in G$ is elliptic in $G$, then $\pi(e)$ is diagonalizable and all the eigenvalues of $\pi(e)$ are of norm 1;

- If $g=e h u$ is the complete multiplicative Jordan decomposition of $g$ in $G$, then $\pi(g)=\pi(e) \pi(h) \pi(u)$ where $\pi(e), \pi(h)$, and $\pi(u)$ mutually commute. Hence $\pi(g)=\pi(e) \pi(h) \pi(u)$ is the complete multiplicative Jordan decomposition of $\pi(g)$ in $\mathrm{GL}_{n}(\mathbb{C})$.

In addition, if $G$ is connected and semisimple, then $\pi(G)$ is generated by

$$
\exp (d \pi(\mathfrak{g}))=\exp ([d \pi(\mathfrak{g}), d \pi(\mathfrak{g})]) \subseteq \exp \mathfrak{s l}_{n}(\mathbb{C})=\mathrm{SL}_{n}(\mathbb{C})
$$

Therefore, $\pi(g) \in \mathrm{SL}_{n}(\mathbb{C})$ and $\pi(g)=\pi(e) \pi(h) \pi(u)$ is the complete multiplicative Jordan decomposition of $\pi(g)$ in $\mathrm{SL}_{n}(\mathbb{C})$. Consequently, we have

Lemma 5. Let $g$ be an element of a connected real semisimple Lie group $G$, and $\pi: G \rightarrow \mathrm{GL}_{n}(\mathbb{C})$ a finite dimensional representation of $G$. If $g=e h u$ is the complete multiplicative Jordan decomposition of $g$ in $G$, then $\pi(g)=\pi(e) \pi(h) \pi(u)$ is the complete multiplicative Jordan decomposition of $\pi(g)$ in $\mathrm{SL}_{n}(\mathbb{C})$.

Now we prove Theorem 3:

Proof of Theorem 3. The necessary part is Theorem 2. It remains to prove the sufficient part. From the assumption, we have $\chi_{\pi}\left(h_{1}\right) \geq$ $\chi_{\pi}\left(h_{2}\right)$ for all finite dimensional representations $\pi$ of $G$. Now suppose 
$h_{1} \nsucceq h_{2}$ in $G$. Then Theorem 1 implies that there exists a finite dimensional representation $\eta: G \rightarrow \mathrm{GL}_{n}(\mathbb{C})$ of $G$ such that $\left|\eta\left(h_{1}\right)\right|<\left|\eta\left(h_{2}\right)\right|$. By Lemma 5 , both $\eta\left(h_{1}\right)$ and $\eta\left(h_{2}\right)$ are hyperbolic in $\mathrm{SL}_{n}(\mathbb{C})$. So they are conjugate to certain diagonal matrices with positive diagonal entries. Using Lemma 4 and the fact that character values are independent of conjugacy, there is a finite dimensional representation $\pi_{m}$ of $\mathrm{GL}_{n}(\mathbb{C})$ with character $\chi_{m}$ such that

$$
\chi_{m}\left(\eta\left(h_{1}\right)\right)<\chi_{m}\left(\eta\left(h_{2}\right)\right) .
$$

Now $\rho:=\pi_{m} \circ \eta$ is a finite dimensional representation of $G$ with character $\chi_{\rho}$ satisfying that

$$
\chi_{\rho}\left(h_{1}\right)=\chi_{m}\left(\eta\left(h_{1}\right)\right)<\chi_{m}\left(\eta\left(h_{2}\right)\right)=\chi_{\rho}\left(h_{2}\right) .
$$

It contradicts our assumption $\chi_{\pi}\left(h_{1}\right) \geq \chi_{\pi}\left(h_{2}\right)$ for all $\pi$.

Note that if $g \in \mathrm{SL}_{n}(\mathbb{C})$ has the complete multiplicative Jordan decomposition $g=e(g) h(g) u(g)$ in $\mathrm{SL}_{n}(\mathbb{C})$, then the hyperbolic component $h(g)$ of $g$ is diagonalizable and its eigenvalues are equal to the eigenvalue moduli of $g$.

Given a finite dimensional representation $\pi$ of $G$ with character $\chi_{\pi}$, let us denote by $\left|\chi_{\pi}\right|(g)$ the sum of eigenvalue moduli of $\pi(g)$ for $g \in G$. Then, from the above observation, we have

$$
\left|\chi_{\pi}\right|(g):=\operatorname{tr}(h(\pi(g))) .
$$

Then Theorem 3 can be extended to an equivalent condition for the partial ordering on all elements of $G$.

Corollary 6. Let $G$ be a connected real semisimple Lie group and $g_{1}, g_{2} \in G$. Then $g_{1} \geq g_{2}$ in $G$ if and only if $\left|\chi_{\pi}\right|\left(g_{1}\right) \geq\left|\chi_{\pi}\right|\left(g_{2}\right)$ for all finite dimensional representations $\pi$ of $G$.

Proof. By the definitions (3) and (4) of the partial order, $g_{1} \geq g_{2}$ in $G$ if and only if $h\left(g_{1}\right) \geq h\left(g_{2}\right)$ in $G$; if and only if $\chi_{\pi}\left(h\left(g_{1}\right)\right) \geq \chi_{\pi}\left(h\left(g_{2}\right)\right)$ for all finite dimensional representations $\pi$ of $G$ (Theorem 3), namely

$$
\operatorname{tr}\left(\pi\left(h\left(g_{1}\right)\right)\right) \geq \operatorname{tr}\left(\pi\left(h\left(g_{2}\right)\right)\right)
$$

According to Lemma 5, $\pi(h(g))=h(\pi(g))$ for all $g \in G$. Thus $\operatorname{tr}\left(\pi\left(h\left(g_{1}\right)\right)\right) \geq \operatorname{tr}\left(\pi\left(h\left(g_{2}\right)\right)\right)$ if and only if $\operatorname{tr}\left(h\left(\pi\left(g_{1}\right)\right)\right) \geq \operatorname{tr}\left(h\left(\pi\left(g_{2}\right)\right)\right)$, namely $\left|\chi_{\pi}\right|\left(g_{1}\right) \geq\left|\chi_{\pi}\right|\left(g_{2}\right)$.

For a finite dimensional representation $\pi: G \rightarrow \mathrm{GL}_{n}(\mathbb{C})$, we denote by

$$
\lambda_{\pi}^{(1)}(g) \geq \lambda_{\pi}^{(2)}(g) \geq \cdots \geq \lambda_{\pi}^{(n)}(g)>0
$$


the eigenvalue moduli of $\pi(g)$ in non-increasing order for $g \in G$, that is, $\lambda_{\pi}^{(1)}(g) \geq \cdots \geq \lambda_{\pi}^{(n)}(g)$ are the eigenvalues of $h(\pi(g))=\pi(h(g))$. Then Theorem 1 says that $g_{1} \geq g_{2}$ in $G$ if and only if $\lambda_{\pi}^{(1)}\left(g_{1}\right) \geq \lambda_{\pi}^{(1)}\left(g_{2}\right)$ for all finite dimensional representations $\pi$ of $G$, and Corollary 6 says that $g_{1} \geq g_{2}$ in $G$ if and only if $\sum_{i=1}^{n} \lambda_{\pi}^{(i)}\left(g_{1}\right) \geq \sum_{i=1}^{n} \lambda_{\pi}^{(i)}\left(g_{2}\right)$ for all finite dimensional representations $\pi: G \rightarrow \mathrm{GL}_{n}(\mathbb{C})$. In general, we have the following result.

Theorem 7. Let $G$ be a connected real semisimple Lie group and $g_{1}, g_{2} \in G$.

(1) If $g_{1} \geq g_{2}$ in $G$, then for every finite dimensional representation $\pi: G \rightarrow \mathrm{GL}_{n}(\mathbb{C})$, the following inequalities hold for $k=1, \cdots, n$ :

$$
\prod_{i=1}^{k} \lambda_{\pi}^{(i)}\left(g_{1}\right) \geq \prod_{i=1}^{k} \lambda_{\pi}^{(i)}\left(g_{2}\right), \quad \sum_{i=1}^{k} \lambda_{\pi}^{(i)}\left(g_{1}\right) \geq \sum_{i=1}^{k} \lambda_{\pi}^{(i)}\left(g_{2}\right) .
$$

(2) Fix $k \in \mathbb{Z}^{+}$. For every finite dimensional representation $\pi$ : $G \rightarrow \mathrm{GL}_{n}(\mathbb{C})$ with $n \geq k$, if we have

$$
\sum_{i=1}^{k} \lambda_{\pi}^{(i)}\left(g_{1}\right) \geq \sum_{i=1}^{k} \lambda_{\pi}^{(i)}\left(g_{2}\right)
$$

then $g_{1} \geq g_{2}$ in $G$.

(3) Fix $k \in \mathbb{Z}^{+}$. For every finite dimensional representation $\pi$ : $G \rightarrow \mathrm{GL}_{n}(\mathbb{C})$ with $n \geq k$, if we have

$$
\prod_{i=1}^{k} \lambda_{\pi}^{(i)}\left(g_{1}\right) \geq \prod_{i=1}^{k} \lambda_{\pi}^{(i)}\left(g_{2}\right)
$$

then $g_{1} \geq g_{2}$ in $G$.

Proof.

(1) Suppose that $g_{1} \geq g_{2}$ in $G$. Given any finite dimensional representation $\pi: G \rightarrow \mathrm{GL}_{n}(\mathbb{C})$, let $\rho_{k}: \mathrm{GL}_{n}(\mathbb{C}) \rightarrow \mathrm{GL}\left(\wedge^{k} \mathbb{C}^{n}\right)$ denote the fundamental representation of $\mathrm{GL}_{n}(\mathbb{C})$ on the $k$-th exterior power of $\mathbb{C}^{n}, k=1, \cdots, n$. Then by Theorem 1 ,

$$
\left|\rho_{k}\left(\pi\left(g_{1}\right)\right)\right| \geq\left|\rho_{k}\left(\pi\left(g_{2}\right)\right)\right|, \quad k=1, \cdots, n-1,
$$

and by $\pi(G) \subseteq \mathrm{SL}_{n}(\mathbb{C})$,

$$
\left|\rho_{n}\left(\pi\left(g_{1}\right)\right)\right|=\left|\operatorname{det}\left(\pi\left(g_{1}\right)\right)\right|=1=\left|\operatorname{det}\left(\pi\left(g_{2}\right)\right)\right|=\left|\rho_{n}\left(\pi\left(g_{2}\right)\right)\right| .
$$


Equivalently,

$$
\begin{aligned}
& \prod_{i=1}^{k} \lambda_{\pi}^{(i)}\left(g_{1}\right) \geq \prod_{i=1}^{k} \lambda_{\pi}^{(i)}\left(g_{2}\right), \quad k=1,2, \cdots, n-1, \\
& \prod_{i=1}^{n} \lambda_{\pi}^{(i)}\left(g_{1}\right)=\prod_{i=1}^{n} \lambda_{\pi}^{(i)}\left(g_{2}\right)=1 .
\end{aligned}
$$

So the vector of eigenvalue moduli of $\pi\left(g_{1}\right)$ multiplicatively majorizes that of $\pi\left(g_{2}\right)$. Moreover, multiplicative majorization of vectors with real positive numbers implies additive majorization [1, Example II.3.5(vii)]. Hence

$$
\sum_{i=1}^{k} \lambda_{\pi}^{(i)}\left(g_{1}\right) \geq \sum_{i=1}^{k} \lambda_{\pi}^{(i)}\left(g_{2}\right), \quad k=1,2, \cdots, n .
$$

(2) Fix $k \in \mathbb{Z}^{+}$. Let $\pi: G \rightarrow \mathrm{GL}_{n}(\mathbb{C})$ be an arbitrary finite dimensional representation of $G$. Let $k \pi$ denote the representation formed by taking the direct sum of $k$ copies of $\pi$. Then the representation space of $k \pi$ has dimension $k n \geq k$. By assumption,

$$
k \lambda_{\pi}^{(1)}\left(g_{1}\right)=\sum_{i=1}^{k} \lambda_{k \pi}^{(i)}\left(g_{1}\right) \geq \sum_{i=1}^{k} \lambda_{k \pi}^{(i)}\left(g_{2}\right)=k \lambda_{\pi}^{(1)}\left(g_{2}\right) .
$$

So $\left|\pi\left(g_{1}\right)\right| \geq\left|\pi\left(g_{2}\right)\right|$. Thus $g_{1} \geq g_{2}$ in $G$ by Theorem 1 .

(3) The proof is similar to that of (2).

\section{REFERENCES}

[1] R. Bhatia, Matrix Analysis, Springer, New York, 1997.

[2] S. Helgason, Differential Geometry, Lie Groups, and Symmetric Spaces, American Mathematical Society, 2001.

[3] B. Kostant, On convexity, the Weyl group and the Iwasawa decomposition, Ann. Sci. École Norm. Sup. (4), 6 (1973) 413-455.

Department of Mathematics and Statistics, Auburn University, AL 36849-5310, USA

E-mail address: huanghu@auburn.edu

Department of Mathematics, The University of Arizona, Tucson, AZ 85721-0089, USA

E-mail address: sangjib@math.arizona.edu 